\title{
Comparison of survival rates between patients treated with transcatheter arterial chemoembolization and hepatic resection for solitary hepatocellular carcinoma
}

\author{
YASUTAKA BABA ${ }^{1}$, SADAO HAYASHI $^{1}$, KAZUTO UENO $^{1}{ }^{2}$ MASAYUKI NAKAJO $^{1}$, SHINICHI UENO ${ }^{2}$, \\ FUMITAKE KUBO ${ }^{2}$, YOSHIROU BABA ${ }^{4}$, MASAHIRO HAMANOUE ${ }^{5}$, SUSUMU HASEGAWA ${ }^{3}$, \\ HIROHITO TSUBOUCHI $^{3}$ and YASUJI KOMORIZONO ${ }^{6}$
}

\begin{abstract}
Departments of ${ }^{1}$ Radiology, ${ }^{2}$ First Surgery, and ${ }^{3}$ Second Internal Medicine, Graduate School of Medical and Dental Sciences, Kagoshima University; Departments of ${ }^{4}$ Internal Medicine and ${ }^{5}$ Surgery, Kagoshima Kouseiren Hospital;

${ }^{6}$ Department of Hepatology, Nanpuh Hospital, Kagoshima, Japan
\end{abstract}

Received April 20, 2010; Accepted June 30, 2010

DOI: 10.3892/ol_00000161

\begin{abstract}
The present study aimed to retrospectively compare the survival rates between patients treated with transcatheter arterial chemoembolization and hepatic resection for solitary hepatocellular carcinoma (HCC). According to our database, derived from three affiliated hospitals, the inclusion criteria for this study were: solitary HCC [Child-Pugh class A and International Union Against Cancer (UICC) stage T1-3NOM0] treated between July 1990 and October 2001. Subsequently, hepatic resection (149 patients) as well as chemoembolization (102 patients) groups were selected. Following stratification according to tumor stage [UICC, Cancer of the Liver Italian Program (CLIP) and Milan criteria], survival rates were compared between the treatment groups. Survival rates were calculated using the Kaplan-Meier method. Age, gender and size of the HCC did not differ significantly between the groups. Moreover, no significant difference in the survival rates (average hepatic resection, 58.9 months; average chemoembolization, 45 months; $\mathrm{P}=0.1697$ ) was observed between the groups. In the subgroup analysis, according to tumor stage, the survival rate was significantly higher for the hepatic resection group than for the chemoembolization group in the UICC T3N0M0 $(\mathrm{P}=0.017)$ subgroup. However, no significant differences in survival rates were observed between the hepatic resection and chemoembolization groups for UICC T1 (P=0.7329), T2N0M0 $(\mathrm{P}=0.5741)$, CLIP0 ( $\mathrm{P}=0.3593), \quad$ CLIP1-2 $\quad(\mathrm{P}=0.3287)$ and within $(<5 \mathrm{~cm} ; \mathrm{P}=0.4429)$ and beyond Milan criteria $(>5 \mathrm{~cm}$; $\mathrm{P}=0.4003)$ subgroups. Chemoembolization is as effective as
\end{abstract}

Correspondence to: Dr Yasutaka Baba, Department of Radiology, Graduate School of Medical and Dental Sciences, Kagoshima University, 8-35-1 Sakuragaoka, Kagoshima-shi, Kagoshima 890-8520, Japan

E-mail: yasutaka@m3.kufm.kagoshima-u.ac.jp

Key words: solitary hepatocellular carcinoma, chemoembolization, hepatic resection, survival hepatic resection in treating solitary $\mathrm{HCC}$ in subpopulations with UICC T1-2N0M0 or CLIP 0-2 HCC or Milan criteria and adequate liver function. In the subgroup with UICC T3NOM0 $\mathrm{HCC}$, hepatic resection is superior to chemoembolization.

\section{Introduction}

Primary liver cancer is one of the most common malignancies that occurs worldwide, and the vast majority of primary liver cancers are hepatocellular carcinoma (HCC) (1). Numerous studies have examined survival in patients with HCC treated by transcatheter arterial chemoembolization (TACE), with chemoembolization showing no clear benefit to survival (2-5). However, patients receiving chemoembolization in these studies included cases with unresectable $\mathrm{HCC}$ and poor liver function.

Solitary HCC with good liver function is usually treated by hepatic resection, but not chemoembolization. However, a small number of studies have described the results of chemoembolization for patients with resectable HCC and good liver function (6).

Findings of this study showed survival rates for patients with resectable HCC who received chemoembolization in comparison to those of HCC patients who underwent hepatic resection. To reduce selection bias from our database, patients selected had solitary HCC and liver function of Child-Pugh A or B and were stratified according to the Cancer of the Liver Italian Program (CLIP) (7), the International Union Against Cancer (UICC) T factor (8) and the Milan criteria (9).

\section{Materials and methods}

Patients. A total of 1,387 patients with newly diagnosed HCC, admitted to three hospitals and treated from July 1990 to October 2001, were studied. According to this database, patients treated with hepatic resection or chemoembolization were recruited. Inclusion criteria were: i) solitary HCC; ii) Child-Pugh class A; and iii) UICC stage T1-3NOMO (8). $\mathrm{T}$ factors in this study were defined as: $\mathrm{T} 1$, solitary tumor 
Table I. Characteristics of the HCC patients.

\begin{tabular}{|c|c|c|c|}
\hline \multirow[t]{2}{*}{ Characteristics } & \multicolumn{2}{|c|}{ Treatment } & \multirow[t]{2}{*}{$\mathrm{P}$-value } \\
\hline & Hepatic resection & TACE & \\
\hline No. of patients & 149 & 102 & \\
\hline Age, years & & & 0.1000 \\
\hline Range & $21-84$ & $37-82$ & \\
\hline Mean & 63.8 & 61.9 & \\
\hline Gender & & & 0.4500 \\
\hline Male/female & $108 / 41$ & $79 / 23$ & \\
\hline TNM classification, & $(\%)$ & & 0.9283 \\
\hline I & 28 (19) & 19 (19) & \\
\hline II & $103(69)$ & $69(68)$ & \\
\hline III & $18(12)$ & $14(13)$ & \\
\hline Tumor size, n (\%) & & & 0.1805 \\
\hline$<2 \mathrm{~cm}$ & $24(16)$ & $15(15)$ & \\
\hline $2-5$ & $91(61)$ & $53(52)$ & \\
\hline $5-10$ & $34(23)$ & $34(33)$ & \\
\hline $\begin{array}{l}\text { Portal vein } \\
\text { involvement, } \mathrm{n}(\%)\end{array}$ & & & 0.8705 \\
\hline $\mathrm{Vp} 0$ & $131(88)$ & $92(90)$ & \\
\hline $\mathrm{Vp} 1$ & $12(8)$ & $6(6)$ & \\
\hline $\mathrm{Vp} 2$ & 4 (3) & $2(2)$ & \\
\hline Vp3 & $2(1)$ & $2(2)$ & \\
\hline CLIP score, n (\%) & & & 0.5452 \\
\hline 0 & $83(56)$ & $52(51)$ & \\
\hline 1 & $54(36)$ & 40 (39) & \\
\hline 2 & $10 \quad(7)$ & $10(10)$ & \\
\hline 3 & 2 (1) & $0 \quad(0)$ & \\
\hline
\end{tabular}

TACE, transcatheter arterial chemoembolization; CLIP, Cancer of the Liver Italian Program.

without vascular invasion; T2, solitary tumor with vascular invasion or multiple tumors, none of which were $>5 \mathrm{~cm}$ in maximum diameter; T3, multiple tumors of $>5 \mathrm{~cm}$ or tumors involving a major branch of the portal or hepatic vein; and T4, tumors with direct invasion of adjacent organs other than the gallbladder or with perforation of visceral peritoneum. The degree of portal vein involvement was classified as: $\mathrm{Vp} 0$, no involvement of the portal vein; $\mathrm{Vp} 1$, involvement of the third or more distal branch of the left or right portal vein; Vp2, involvement of the second branch of the portal vein; and Vp3, involvement of the first branch or trunk of the portal vein.

The subjects were divided into three groups according to portal vein involvement ( Vp0-1, Vp2 and Vp3). Subjects comprised 187 men and 64 women, with a mean age of 63 years (range 21-84). A total of 164 patients were hepatitis $C$ viruspositive $(65 \%)$ and 43 patients were hepatitis $\mathrm{B}$ virus-positive (17\%). Hepatitis B and C were positive in 2 patients (1\%) and negative in 42 patients (17\%). HCC was diagnosed based on findings obtained from ultrasonography, biphasic dynamic computed tomography (CT), dynamic magnetic resonance imaging (MRI) and angiography, and/or pathologically by biopsy specimens. Serum $\alpha$-fetoprotein or protein induced by vitamin $\mathrm{K}$ absence or antagonist-II (PIVKAII) was also
Table II. Comparison of the two groups of hepatic resection and TACE in demographics.

\begin{tabular}{lccc}
\hline & $\begin{array}{c}\text { Hepatic } \\
\text { resection } \\
(\mathrm{n}=149)\end{array}$ & TACE & P-value \\
& & $\mathrm{n}=102)$ & \\
\hline Etiology & 25 & 18 & \\
Hepatitis B & 101 & 63 & \\
Hepatitis C & 1 & 1 & \\
Hepatitis B and C & 22 & 20 & \\
Non-B, non-C & $3.9(2.5-5.1)$ & $3.9(1.7-6.7)$ & 0.9148 \\
Platelet count (x10,000) & $13.7(2.8-123)$ & $13.7(3.8-35.9)$ & 0.9700 \\
Albumin (g/dl) & $86(43-108)$ & $86(59-108)$ & 0.7698 \\
Prothrombin time (sec) & $3.6(1.0-8.5)$ & $3.9(1.0-9.7)$ & 0.1109 \\
Tumor size (cm) & & & \\
\hline
\end{tabular}

TACE, transcatheter arterial chemoembolization.

determined. The mean tumor size was $3.7 \mathrm{~cm}$ (range 1-9.7). Informed consent was obtained from all patients after information was provided concerning the HCC and the two treatments (chemoembolization and hepatic resection). As a result, 149 patients received hepatic resection and 102 patients, who declined hepatic resection, received chemoembolization. Age, gender, size of HCC and background patient characteristics did not differ significantly between the hepatic resection and TACE groups (Tables I and II).

Chemoembolization (10). Hepatic arteriography was performed using Seldginger's method. After arterial access, diagnostic arteriography was performed to evaluate hepatic arterial and portal venous anatomy. Following the study of CT during arterial portography to assess whether the liver tumor was solitary, superselective catheterization was performed in tumor-feeding vessels. The coaxial catheter system was used to perform chemoembolization (Tracker-18 infusion catheter or Renegade; Boston Scientific, Fremont, CA, USA). The chemotherapeutic agent (epirubicin; Kyowa Hakko Kogyo, Tokyo, Japan) was dissolved in a solution of non-ionic watersoluble contrast medium and saline solution and mixed with lipiodol (Laboratoire Andre Guerbet, Paris, France). The dose of iodized oil and anticancer drugs was determined on the basis of tumor size, hepatic function, renal function and blood chemistry data. After the microcatheter tip was placed in the tumor-feeding vessel without stopping blood inflow, the chemotherapeutic agent was injected. Following confirmation of little or no visualization of tumor staining on arteriography, gelfoam particles (Gelfoam; Upjohn, Kalamazoo, MI, USA) were injected into tumor vessels as an embolizing agent (6,11-16). CT was performed at 7-10 days and 1 month after treatment, and subsequently every 2-3 months. If recurrent lesions appeared on the follow-up CT, chemoembolization was repeated.

Hepatic resection. Hepatic resection was performed for 149 patients. Methods of hepatic resection were: subsegment- 


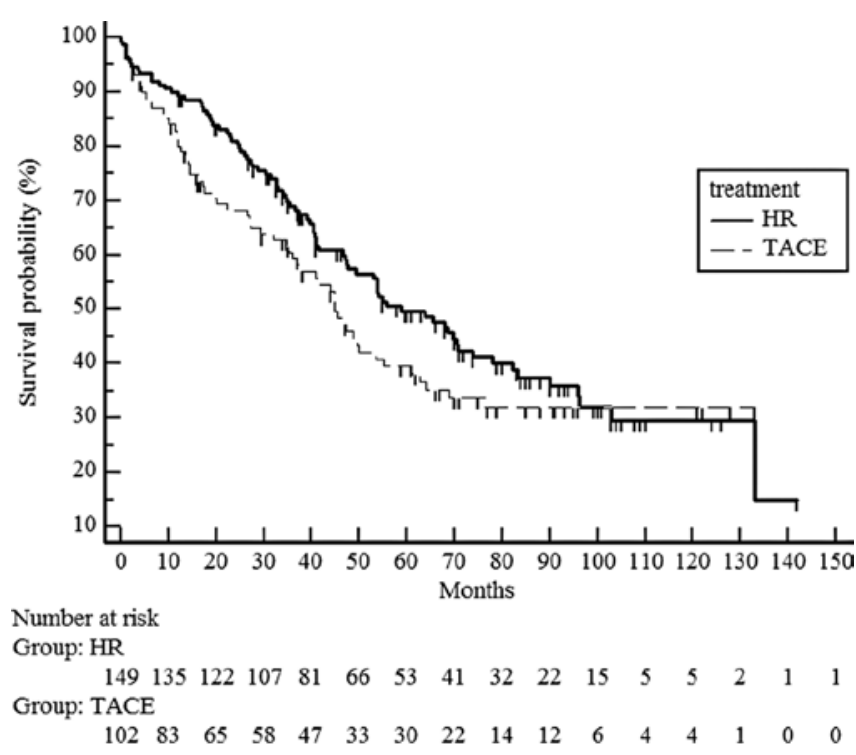

Figure 1. Overall patient survival. HR, hepatic resection; TACE, transcatheter arterial chemoembolization.

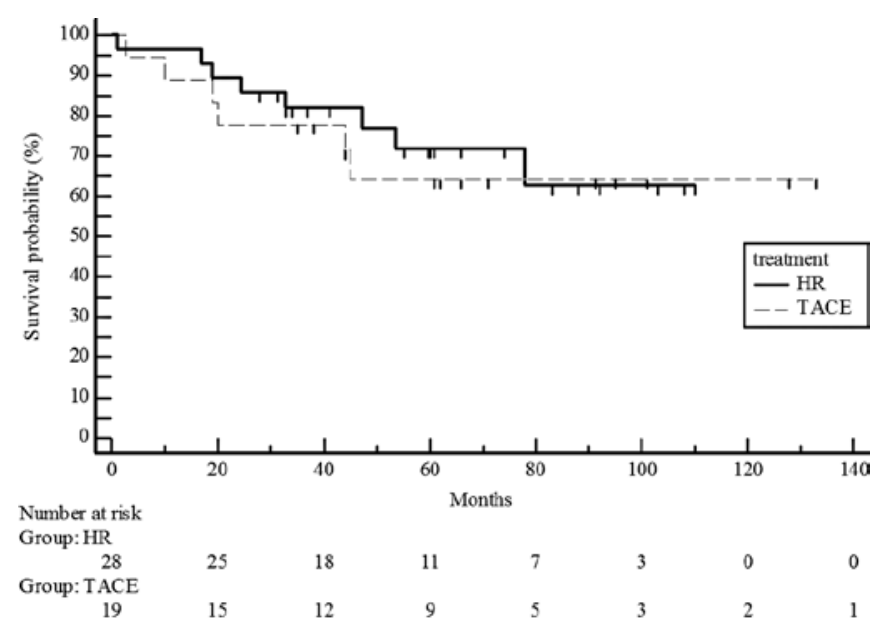

Figure 2. Survival rates for UICC T1 stage patients. HR, hepatic resection; TACE, transcatheter arterial chemoembolization.

ectomy, 111 patients; segmentectomy, 25 patients; lobectomy, 11 patients; and extended lobectomy, 2 patients. No patients succumbed to or presented with complications related to the hepatic resection.

Statistical analysis. The main end-point (survival from initial treatment) was evaluated for the hepatic resection and chemoembolization groups using the Kaplan-Meier method and compared statistically by log-rank testing. According to UICC, CLIP scores and Milan criteria, patients were stratified, and survival rates were compared between the treatment groups according to each stratification. Statistical analysis was carried out using the Student's t-test for continuous variables and a Chi-square test for categorical variables with commercially available software packages (MedCalc Version 9.5.1.0; MedCalc Software, Mariakerke, Belgium). A two-tailed P-value $(\mathrm{P}<0.05)$ was considered significant.

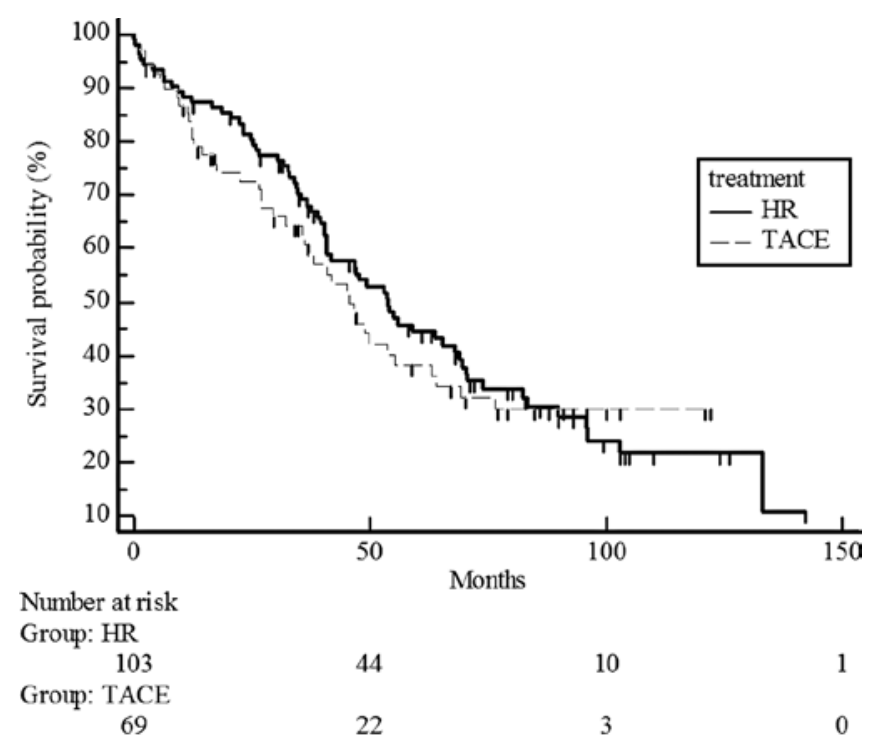

Figure 3. Survival rates for UICC T2 stage patients. HR, hepatic resection; TACE, transcatheter arterial chemoembolization.

\section{Results}

Survival analysis of total hepatic resection and chemoembolization groups. By October 2001, 79 of the 149 patients with hepatic resection treatment and 54 of the 102 patients with chemoembolization were deceased. No significant difference in the causes of death was noted between the two treatment groups, with the majority of deaths resulting from liver failure, including hepatic encephalopathy and spontaneous bacterial peritonitis, varix bleeding or progression of the tumor itself. During the follow-up period of chemoembolization, radiofrequency ablation (RFA) or percutaneous ethanol injection therapy (PEIT) was performed in 4 patients $(3 \%)$ and repeated chemoembolization was performed in 24 patients (24\%). During the follow-up period of hepatic resection, chemoembolization and PEIT were performed in 45 patients (30\%; 40 and 5 patients, respectively).

In the chemoembolization group, 1 patient was lost to follow-up and was censored, while no patients were lost to follow-up in the hepatic resection group. The median duration of follow-up was 47.4 months (Fig. 1). Median survival time was 51 months in the hepatic resection group and 41 months in the chemoembolization group. No significant difference in survival was noted between the hepatic resection and chemoembolization groups (median survival time 58.9 vs. 45 months) $(\mathrm{P}=0.1697)$ (Fig. 1).

Subgroup survival analysis of hepatic resection and chemoembolization groups

UICC T stage. Survival rates did not differ significantly for UICC T1 stage patients in the hepatic resection and chemoembolization groups $(\mathrm{P}=0.7329$; estimated 5-year survival rate, 70 vs. 65\%) (Fig. 2) and T2 ( $\mathrm{P}=0.5741$; estimated 5-year survival rate, 44 vs. 38\%) (Fig. 3). However, survival rates were significantly different for UICC T3, with higher rates in the hepatic resection group than in the chemoembolization 


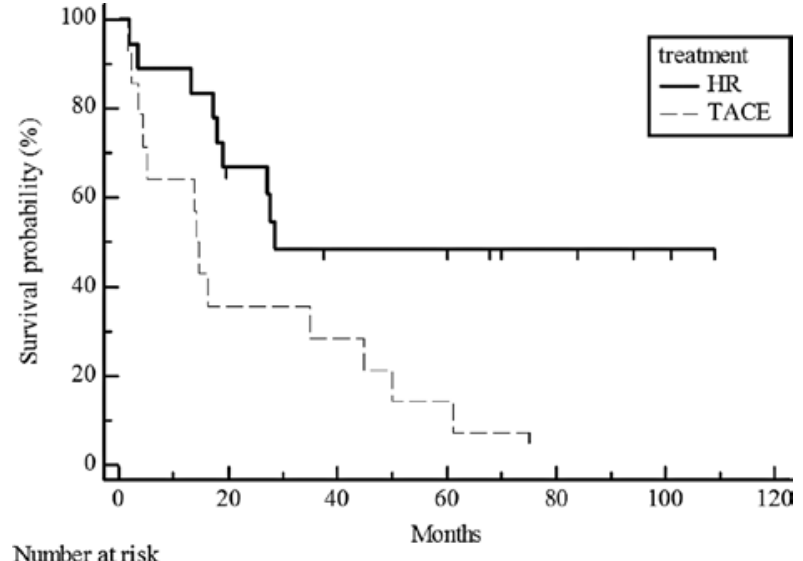

Number at ris

Group: HR

$\begin{array}{ccccccc}18 & 11 & 7 & 6 & 4 & 2 & 1 \\ \text { Group: TACE } & & & 2 & 0 & 0 & 0\end{array}$

Figure 4. Survival rates for UICC T3 stage patients. HR, hepatic resection; TACE, transcatheter arterial chemoembolization.

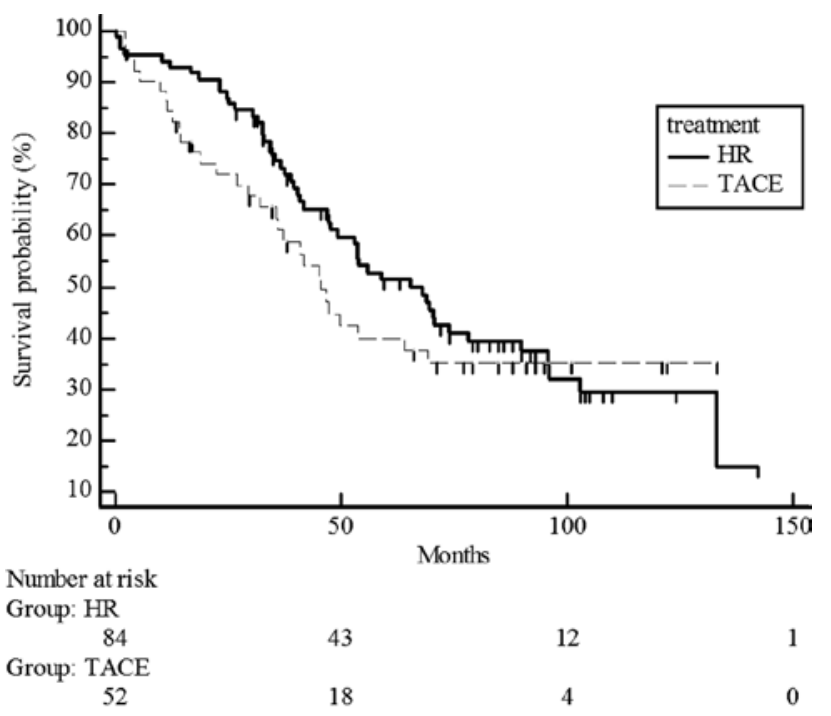

Figure 5. Survival rates for patients in the CLIP 0 group. HR, hepatic resection; TACE, transcatheter arterial chemoembolization.

group ( $\mathrm{P}=0.017$; estimated 5-year survival rate, 48 vs. $14 \%)$ (Fig. 4).

CLIP score. Survival rates did not differ significantly between the hepatic resection and chemoembolization groups in CLIP 0 ( $\mathrm{P}=0.3593$; estimated 5-year survival rate, 51 vs. $40 \%)$ (Fig. 5) and in the CLIP 1-2 groups $(\mathrm{P}=0.3287$; estimated 5-year survival rate, 47 vs. $39 \%$ ) (Fig. 6).

Milan criteria. Survival rates did not differ significantly between the hepatic resection and chemoembolization groups within Milan criteria $(<5 \mathrm{~cm}, \mathrm{P}=0.4429$; estimated 5-year survival rate, 53 vs. 43\%) (Fig. 7) and beyond Milan criteria ( $>5 \mathrm{~cm}, \mathrm{P}=0.4$; estimated 5 -year survival rate, 39 vs. $30 \%$ ) (Fig. 8).

Patient complications. In the chemoembolization group, no relevant post-embolization complication, including death related to chemoembolization, was reported. Post-embolization syndrome, including mild abdominal pain and fever, was

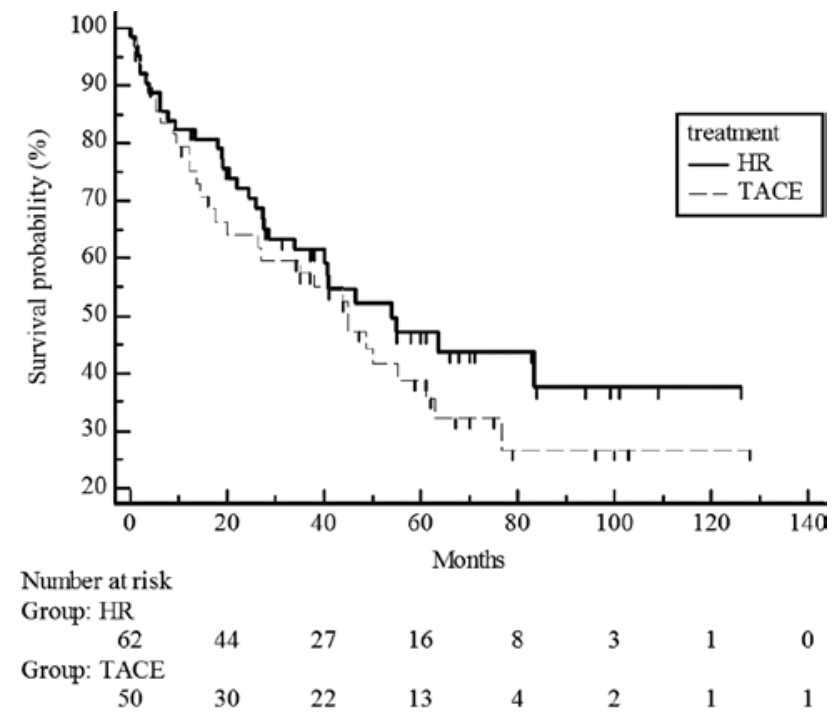

Figure 6. Survival rates for patients in the CLIP 1-2 group. HR, hepatic resection; TACE, transcatheter arterial chemoembolization.

common and treated with anti-inflammatory drugs. On the other hand, four patients succumbed (2.7\%) within 30 days after surgery in the hepatic resection group. However, it was not evident that there was a direct relationship between hepatic resection and death.

\section{Discussion}

Previous reports showed that the treatment modalities offering a cure of HCC include surgical resection (17,18), PEIT (19), RFA (20) and liver transplantation (21). However, chemoembolization has yet to be considered as a curative treatment of choice for HCC. The reason for this is that numerous studies have been unable to demonstrate any improvement in survival for the chemoembolization treatment of $\operatorname{HCC}(2,22)$.

Chemoembolization involves mixing iodized oil and one or more anticancer drugs, such as doxorubicin hydrochloride, epirubicin hydrochloride, mitomycin C, cisplatin, neocarzinostatin or floxuridine; injecting the mixture into tumor-feeding vessels; and embolizing the vessels with gelatin sponges $(3,6,11,14-16,23-33)$. In our series, the main anticancer drug used in chemoembolization was epirubicin hydrochloride. According to the latest nationwide report by the Liver Cancer Study Group of Japan, anticancer drugs used for chemoembolization in Japan include doxorubicin hydrochloride, epirubicin hydrochloride and cisplatin (33). However, strict dose criteria for anticancer drugs and lipiodol have yet to be determined. The objective of chemoembolization is to accumulate lipiodol in the liver tumor as compactly as possible $(4,6,13,15,24,34)$. Almost all patients in our study undertook CT approximately 1 week after chemoembolization. When the accumulation of lipiodol in the tumor was insufficient, additional chemoembolization was performed. Lee et al reported favorable survival rates for patients with $\mathrm{HCC}$ who received chemoembolization when lipiodol was compactly retained (6).

Chemoembolization has been used as a palliative therapy for unresectable HCC. Previous reports showed that eligible candidates for chemoembolization are patients with unresect- 


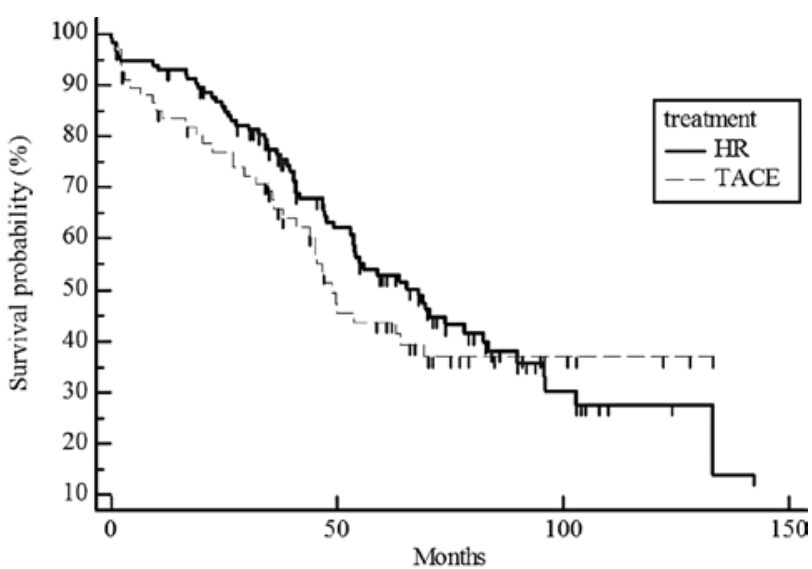

Number at risk
Group: HR
114
Group: TACE
68

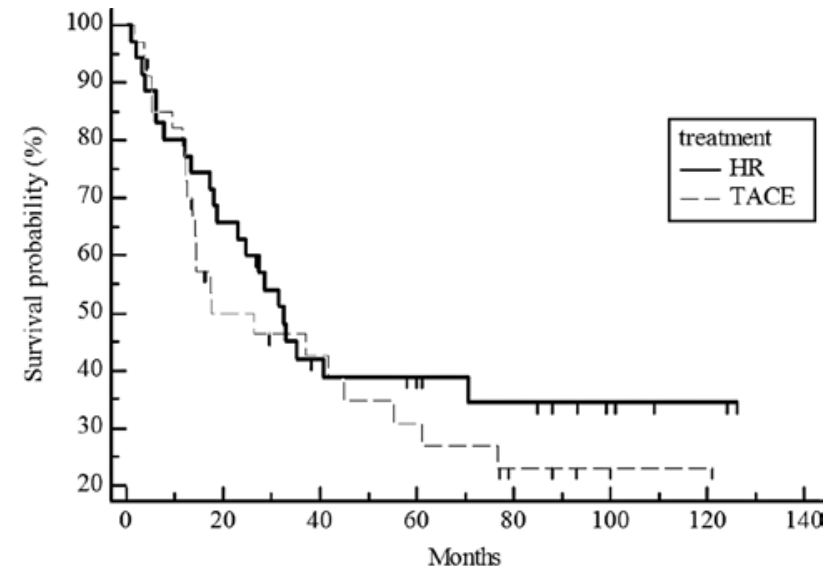

Number at risk Group: HR

$\begin{array}{cccccccc}35 & 23 & 13 & 10 & 8 & 4 & 2 & 1 \\ \text { Group: TACE } & & & & & & & \\ 34 & 14 & 11 & 8 & 4 & 1 & 1 & 0\end{array}$

Figure 7. Survival rates for patients within Milan criteria $(>5 \mathrm{~cm})$. HR, hepatic resection; TACE, transcatheter arterial chemoembolization.

able HCC and poor liver function, multiple liver tumors $(>3)$ or large tumor size $(>10 \mathrm{~cm})(4,5,16,22,30,32,35)$.

An initial randomized controlled trial of chemoembolization did not identify superior survival by chemoembolization compared to palliative therapies $(2,22,36,37)$. However, previous randomized control trials showed that chemoembolization is superior to symptomatic treatment in terms of the 2-year survival rate $(38,39)$.

Resected specimens following chemoembolization have shown a high correlation between complete retention of lipiodol in the tumor and pathological necrosis $(11,14,24,34,40)$. In those studies, patients had good liver function and a solitary liver tumor. Therefore, the possibility of a favorable prognosis exists after chemoembolization in selected patients with operable HCC. Choi et al (24) stated that chemoembolization was performed to reduce the possibility of tumor recurrence and to decrease tumor size in operable cases of HCC. Takayasu et al (15) reported a correlation between lipiodol accumulation in the HCC and survival rate. These authors showed that survival rates of 1, 2 and 3 years after TACE were 93.3, 77.1 and 77.1\%, respectively. In comparison, our results demonstrated that survival rates of 1, 2 and 3 years after TACE were 80.9, 68.2 and $59.3 \%$, respectively. However, Takayasu et al used the inclusion criterion for chemoembolization of one main lesion $(<5 \mathrm{~cm})$ associated with no more than two lesions $(<3 \mathrm{~cm})$ (15), and patients with solitary HCC underwent surgery. In addition, few studies have compared hepatic resection and chemoembolization in patients with solitary HCC and good liver function (6).

Numerous criteria have been proposed for an HCC staging system (41-46). Among these criteria, the most frequently used are the Okuda staging system (47), the Child-Pugh staging system (48), tumor node metastasis (TNM) staging (8) and CLIP score (7). Although a number of obstacles and limitations exist with these proposed criteria, Georgiades et al (42) reported Child-Pugh staging as the most accurate of 12 liver staging systems for predicting results in unresectable
Figure 8. Survival rates for patients beyond Milan criteria $(\leq 5 \mathrm{~cm})$. HR, hepatic resection; TACE, transcatheter arterial chemoembolization.

HCC patients. We applied the Child-Pugh staging system, TNM staging and CLIP score as liver staging systems for our patients.

The present results showed that the survival rate did not differ significantly between the hepatic resection and chemoembolization groups for UICC T1-2 HCC, while a significant difference was apparent for UICC T3 HCC. UICC T3 indicates multiple tumors larger than $5 \mathrm{~cm}$ or a tumor involving a major branch of the portal or hepatic veins. Previous reports indicated tumor size, number of tumors, serum $\alpha$-fetoprotein levels, liver function and portal vein involvement as prognostic factors of HCC (16). In our study, no significant differences in the number of tumors and liver function were noted between the two groups. However, portal vein involvement was not thoroughly considered in the T3 HCC subgroup between treatments. Portal vein involvement may thus be one source of survival bias.

The Milan criteria are used in patient selection for liver transplantation (9), which is considered to be the optimal treatment of small HCC. Bridge treatments, including hepatic resection, TACE and RFA, are necessary for patients anticipating organ transplantation $(50,51)$. Roayaie et al (51) reported that patients with $\mathrm{HCC}$ measuring more or equal to $5 \mathrm{~cm}$ achieve long-term survival after liver transplantation combined with TACE. Belghiti et al (50) reported that liver resection prior to liver transplantation does not increase the morbidity nor impair long-term survival following liver transplantation in patients with Milan criteria. Our results showed that there was no significant difference between chemoembolization and hepatic resection in patients both within $(<5 \mathrm{~cm})$ and beyond $(>5 \mathrm{~cm})$ Milan criteria. We suggest that patients eligible for liver transplantation should be managed by hepatic resection or TACE until such time organ transplantation occurs.

Limitations were noted in this study. The study design was retrospective and showed selection bias. Furthermore, the backgrounds of chronic liver damage varied. The majority of background disease was hepatitis $\mathrm{B}$ or $\mathrm{C}$, but the two diseases exhibit different characteristics $(41,45)$. The background bias 
should therefore be considered. In conclusion, chemoembolization appears to be as effective as hepatic resection in treating solitary HCC and in subpopulations with UICC T1-2NOM0 or CLIP 0-2 HCC with adequate liver function. However, hepatic resection is preferable for treating the subgroup of patients with UICC T3NOMO HCC.

\section{References}

1. Okuda K: Hepatocellular carcinoma. J Hepatol 32: 225-237, 2000.

2. Anonymous: A comparison of lipiodol chemoembolization and conservative treatment for unresectable hepatocellular carcinoma: Groupe d' Etude et de Traitement du Carcinome Hepatocellulaire. N Engl J Med 332: 1256-1261, 1995.

3. Achenbach T, Seifert JK, Pitton MB, Schunk K and Junginger T: Chemoembolization for primary liver cancer. Eur J Surg Oncol 28: 37-41, 2002.

4. Dumortier J, Chapuis F, Borson O, et al: Unresectable hepatocellular carcinoma: survival and prognostic factors after lipiodol chemoembolisation in 89 patients. Dig Liver Dis 38: 125-133, 2006.

5. Mondazzi L, Bottelli R, Brambilla G, et al: Transarterial oily chemoembolization for the treatment of hepatocellular carcinoma: a multivariate analysis of prognostic factors. Hepatology 19: 1115-1123, 1994.

6. Lee HS, Kim KM, Yoon JH, et al: Therapeutic efficacy of transcatheter arterial chemoembolization as compared with hepatic resection in hepatocellular carcinoma patients with compensated liver function in a hepatitis B virus-endemic area: a prospective cohort study. J Clin Oncol 10: 4459-4465, 2002.

7. The Cancer of the Liver Italian Program: A new prognostic system for hepatocellular carcinoma: a retrospective study of 435 patients. Hepatology 28: 751-755, 1998.

8. Sobin LH and Wittekind Ch (eds): International Union Against Cancer (UICC). Liver. TNM Classification of Malignant Tumours. 6th edition. John Wiley \& Sons, Inc., New York, 2002.

9. Mazzaferro V, Regalia E, Doci R, et al: Liver transplantation for the treatment of small hepatocellular carcinoma in patients with cirrhosis. N Engl J Med 334: 693-699, 1996.

10. Brown DB, Gould JE, Gervais DA, et al: Transcatheter therapy for hepatic malignancy: standardization of terminology and reporting criteria. J Vasc Interv Radiol 18: 1469-1478, 2007.

11. Higashihara $\mathrm{H}$ and Okazaki M: Transcatheter arterial chemoembolization of hepatocellular carcinoma: a Japanese experience. Hepatogastroenterology 28: 72-78, 2002.

12. Matsuo $\mathrm{N}$, Uchida $\mathrm{H}$, Nishimine $\mathrm{K}$, et al: Segmental transcatheter hepatic artery chemoembolization with iodized oil for hepatocellular carcinoma: antitumor effect and influence on normal tissue. J Vasc Interv Radiol 4: 543-549, 1993.

13. Miyayama S, Matsui O, Yamashiro M, et al: Ultraselective transcatheter arterial chemoembolization with a 2-f tip microcatheter for small hepatocellular carcinomas: relationship between local tumor recurrence and visualization of the portal vein with iodized oil. J Vasc Interv Radiol 18: 365-376, 2007.

14. Nakamura H, Liu T, Hori S, et al: Response to transcatheter oily chemoembolization in hepatocellular carcinoma $3 \mathrm{~cm}$ or less: a study in 50 patients who underwent surgery. Hepatogastroenterology 3: 6-9, 1993.

15. Takayasu K, Muramatsu Y, Maeda T, et al: Targeted transarterial oily chemoembolization for small foci of hepatocellular carcinoma using a unified helical CT and angiography system: analysis of factors affecting local recurrence and survival rates. Am J Roentgenol 176: 681-688, 2001.

16. Ueno K, Miyazono N, Inoue H, Nishida H, Kanetsuki I and Nakajo M: Transcatheter arterial chemoembolization therapy using iodized oil for patients with unresectable hepatocellular carcinoma: evaluation of three kinds of regimens and analysis of prognostic factors. Cancer 172: 1574-1581, 2000.

17. Ikai I, Arii S, Kojiro M, et al: Reevaluation of prognostic factors for survival after liver resection in patients with hepatocellular carcinoma in a Japanese nationwide survey. Cancer 101: 796-802, 2004.
18. Yamamoto J, Kosuge T, Saiura A, et al: Effectiveness of hepatic resection for early-stage hepatocellular carcinoma in cirrhotic patients: subgroup analysis according to Milan criteria. Jpn J Clin Oncol 37: 287-295, 2007.

19. Sung Y, Choi D, Lim H, et al: Long-term results of percutaneous ethanol injection for the treatment of hepatocellular carcinoma in Korea. Korean J Radiol 7: 187-192, 2006.

20. Tateishi R, Shiina S, Teratani T, et al: Percutaneous radiofrequency ablation for hepatocellular carcinoma. An analysis of 1000 cases. Cancer 103: 1201-1209, 2005.

21. Imvrios G, Papanikolaou V, Vrochides D, et al: Liver transplantation outcomes in patients with cirrhosis and hepatocellular carcinoma: experience of a single center in a viral hepatitis endemic area. Transplant Proc 39: 1508-1510, 2007.

22. Bruix J, Llovet J, Castells A, et al: Transarterial embolization versus symptomatic treatment in patients with advanced hepatocellular carcinoma: results of a randomized, controlled trial in a single institution. Hepatology 27: 1578-1583, 1998.

23. Chen MS, Li JQ, Zhang YQ, et al: High-dose iodized oil transcatheter arterial chemoembolization for patients with large hepatocellular carcinoma. World J Gastroenterol 8: 74-78, 2001.

24. Choi B, Kim H, Han J, et al: Therapeutic effect of transcatheter oily chemoembolization therapy for encapsulated nodular hepatocellular carcinoma: CT and pathologic findings. Radiology 182: 709-713, 1992.

25. Eurvilaichit C: Outcome of transcatheter oily chemoembolization in patients with hepatocellular carcinoma. Hepatogastroenterology 51: 20-24, 2004.

26. Hashimoto N, Kawai S, Mikuriya S, et al: Effects of transcatheter arterial chemoembolization with oral chemotherapy on hepatic neoplasms. Cancer Chemother Pharmacol 23: S21-S25, 1989.

27. Kamada K, Nakanishi T, Kitamoto M, et al: Long-term prognosis of patients undergoing transcatheter arterial chemoembolization for unresectable hepatocellular carcinoma: comparison of cisplatin lipiodol suspension and doxorubicin hydrochloride emulsion. J Vasc Interv Radiol 12: 847-854, 2001.

28. Maeda S, Shibata J, Fujiyama S, et al: Long-term follow-up of hepatic arterial chemoembolization with cisplatin suspended in iodized oil for hepatocellular carcinoma. Hepatogastroenterology 50: 809-813, 2003.

29. Okusaka T, Okada S, Ueno H, et al: Transcatheter arterial embolization with zinostatin stimalamer for hepatocellular carcinoma. Oncology 62: 228-233, 2002.

30. Ono Y, Yoshimasu T, Ashikaga R, et al: Long-term results of lipiodol-transcatheter arterial embolization with cisplatin or doxorubicin for unresectable hepatocellular carcinoma. Am J Clin Oncol 23: 564-568, 2000.

31. Shimamura Y, Gunven P, Takenaka Y, et al: Combined peripheral and central chemoembolization of liver tumors. Experience with lipiodol-doxorubicin and gelatin sponge (L-TAE). Cancer 61: 238-242, 1987.

32. Stuart K, Stokes K, Jenkins R, Trey C and Clouse M: Treatment of hepatocellular carcinoma using doxorubicin/ethiodized oil/ gelatin powder chemoembolization. Cancer 72: 3202-3209, 1993.

33. Takayasu K, Arii S, Ikai I, et al: Prospective cohort study of transarterial chemoembolization for unresectable hepatocellular carcinoma in 8510 patients. Gastroenterology 131: 461-469, 2006.

34. Takayasu K, Arii S, Matsuo N, et al: Comparison of CT findings with resected specimens after chemoembolization with iodized oil for hepatocellular carcinoma. Am J Roentgenol 175: 699-704, 2000.

35. O'Suilleabhain CB, Poon RT, Yong JL, Ooi GC, Tso WK and Fan ST: Factors predictive of 5-year survival after transarterial chemoembolization for inoperable hepatocellular carcinoma. $\mathrm{Br}$ J Surg 90: 325-331, 2002.

36. Pelletier G, Ducreux M, Gay F, et al: Treatment of unresectable hepatocellular carcinoma with lipiodol chemoembolization: a multicenter randomized trial. Groupe CHC. J Hepatol 29: 129-134, 1998.

37. Pelletier G, Roche A, Ink O, et al: A randomized trial of hepatic arterial chemoembolization in patients with unresectable hepatocellular carcinoma. J Hepatol 11: 181-184, 1990.

38. Llovet J, Real M, Montana X, et al: Arterial embolisation or chemoembolisation versus symptomatic treatment in patients with unresectable hepatocellular carcinoma: a randomised controlled trial. Lancet 18: 1734-1739, 2002. 
39. Lo CM, Ngan H, Tso WK, et al: Randomized controlled tria of transarterial lipiodol chemoembolization for unresectable hepatocellular carcinoma. Hepatology 35: 1164-1171, 2002.

40. Takayasu K, Moriyama N, Muramatsu Y, et al: Hepatic arterial embolization for hepatocellular carcinoma. Comparison of CT scans and resected specimens. Radiology 150: 661-665, 1984.

41. Kudo M, Chung H, Haji S, et al: Validation of a new prognostic staging system for hepatocellular carcinoma: the JIS score compared with the CLIP score. Hepatology 40: 1396-1405, 2004.

42. Georgiades C, Liapi E, Frangakis C, et al: Prognostic accuracy of 12 liver staging systems in patients with unresectable hepatocellular carcinoma treated with transarterial chemoembolization. J Vasc Interv Radiol 17: 1619-1624, 2006.

43. Kudo M, Chung H and Osaki Y: Prognostic staging system for hepatocellular carcinoma (CLIP score): its value and limitations, and a proposal for a new staging system, the Japan Integrated Staging Score (JIS score). J Gastroenterol 38: 207-215, 2003.

44. Leung T, Tang A, Zee B, et al: Construction of the Chinese University Prognostic Index for hepatocellular carcinoma and comparison with the TNM staging system, the Okuda staging system, and the Cancer of the Liver Italian Program staging system: a study based on 926 patients. Cancer 94: 1760-1769, 2002.

45. Ueno S, Tanabe G, Nuruki K, et al: Prognosis of hepatocellular carcinoma associated with Child class $\mathrm{B}$ and $\mathrm{C}$ cirrhosis in relation to treatment: a multivariate analysis of 411 patients at a single center. J Hepatobiliary Pancreat Surg 9: 469-477, 2002.
46. Ueno S, Tanabe G, Sako K, et al: Discrimination value of the new western prognostic system (CLIP score) for hepatocellular carcinoma in 662 Japanese patients. Cancer of the Liver Italian Program. Hepatology 34: 529-534, 2001.

47. Okuda K, Ohtsuki T, Obata H, et al: Natural history of hepatocellular carcinoma and prognosis in relation to treatment. Study of 850 patients. Cancer 56: 918-928, 1985.

48. Pugh R, Murray-Lyon I, Dawson J, Pietroni M and Williams R: Transection of the oesophagus for bleeding oesophageal varices. Br J Surg 60: 646-669, 1973.

49. Majno PE, Sarasin FP, Mentha G and Hadengue A: Primary liver resection and salvage transplantation or primary liver transplantation or primary liver transplantation in patients with single, small hepatocellular carcinoma and preserved liver function: an outcome-oriented decision analysis. Hepatology 31: 899-906, 2000.

50. Belghiti J, Cortes A, Abdalla EK, et al: Resection prior to liver transplantation for hepatocellular carcinoma. Ann Surg 238: 885-892, 2003.

51. Roayaie S, Frischer JS, Emre SH, et al: Long-term results with multimodal adjuvant therapy and liver transplantation for the treatment of hepatocellular carcinomas larger than 5 centimeters. Ann Surg 235: 533-539, 2002. 